\title{
Comment des pédagogies alternatives peuvent aider à la réussite des étudiants de physique du $7^{\mathrm{er}}$ cycle universitaire
}

Raphaëlle Demeyer $^{(1)}$ et Jérôme Margueron ${ }^{(2)}$ (j.margueron@ipnl.in2p3.fr)

(1) Chargée de mission « Évaluation innovation pédagogique » à l'Université de Lyon

(2) Institut de physique des 2 infinis de Lyon (UMR 5822), Université Claude Bernard Lyon 1 ,

Bâtiment Paul Dirac, 4 rue Enrico Fermi, 69622 Villeurbanne Cedex

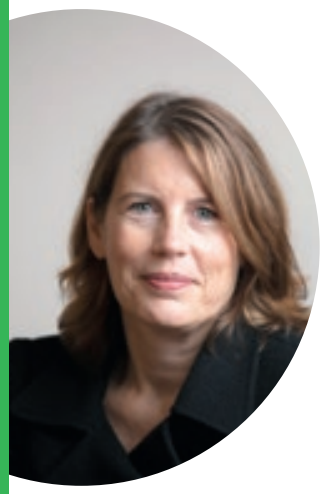

Paula Heron

\section{Biographie}

Paula Heron a obtenu sa thèse en physique théorique à l'Université Western Ontario (Canada) avant de rejoindre l'équipe professorale de physique de l'Université de Washington à Seattle, où elle s'est passionnée pour les sciences de l'éducation.

Elle mène depuis plusieurs années un travail de recherche visant à trouver des solutions pour que les étudiants de licence (undergraduate degree) développent une connaissance plus fine et plus solide des concepts physiques fondamentaux.

Paula Heron est en particulier coauteure du rapport Phys21: Preparing Physics Students for 21 st Century Careers [1], ainsi que de l'ouvrage Tutorials in Introductory Physics [2] (encadré 1, p. 35). Elle est coprésidente du cycle de conférences "Foundations and Frontiers in Physics Education Research", regroupant la communauté des chercheurs dans l'éducation à la physique en Amérique du Nord.
Jérôme Margueron (Q) : Bonjour, Paula. Pouvez-vous revenir sur ce qui motive, selon vous, l'intérêt de moderniser la pédagogie dans le domaine de l'enseignement supérieur en physique?

Paula Heron (PH) : La physique est certes une science exacte, mais on s'aperçoit que les jeunes inferent, à partir des phénomènes physiques qui les entourent, des connaissances intuitives qui peuvent ancrer des erreurs de compréhension plus tard dans leur cursus. Ainsi, les notions d'accélération et de vitesse sont quelquefois confuses, ce qui peut mener à des incompréhensions. Celles-ci peuvent se poursuivre lorsqu'on aborde les concepts de potentiel et d'énergie potentielle, par exemple.

Les cours magistraux classiques ne permettent que rarement de remettre en cause des préjugés bien ancrés, et ce malgré tous les efforts de clarification du message et d'apports de preuves expérimentales des enseignants.

Les professeurs de physique le savent bien, et ont depuis longtemps engagé d'autres façons d'enseigner, en particulier dans l'enseignement secondaire. Mais force est de constater que les difficultés persistent dans les premières années du supérieur. Le moindre point non traité - ou moins bien traité - dans les programmes du collège (middle school) et du lycée (high school) aboutit à de gros soucis d'apprentissage à l'université. Là, les cours s'accélèrent mais les incompréhensions s'enracinent et mènent, hélas, certains étudiants à l'échec. C'est un problème pour tous les étudiants, mais en particulier pour ceux qui sortent de milieux moins favorisés, de zones scolaires en difficulté. La réduction du "gap" social et culturel est un enjeu sur lequel nous travaillons beaucoup.

Q : Quels sont les concepts sur lesquels s'appuie votre méthode des "Tutorials" ?

PH : La recherche dans les sciences de l'éducation apporte de multiples éclairages sur la façon dont les étudiants apprennent, autant en matière de mémorisation à long terme qu'en ce qui concerne la capacité à appliquer leurs savoirs.

Ainsi, il a été prouvé par de nombreux travaux de recherche qu'amener l'étudiant à " reconstruire " le concept lui-même, pas-à-pas, est une méthode extrêmement efficace, très complémentaire des cours 


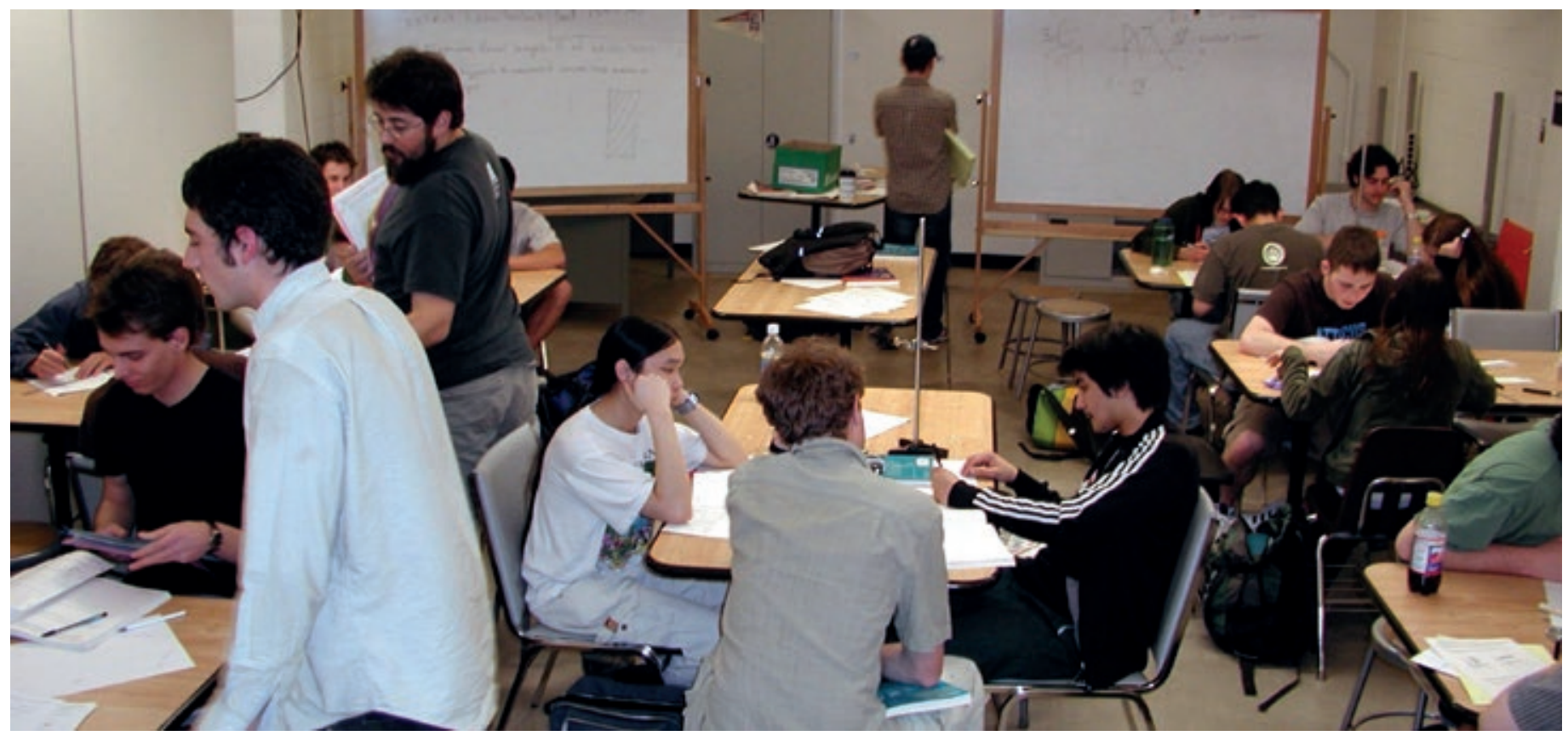

1. Séance de "Tutorial". Petits groupes d'étudiants échangeant leurs idées autour d'une table.

magistraux et des travaux pratiques. Bien entendu, il faut que le chemin pour y arriver soit bien balisé, car l'étudiant doit réussir à raisonner de lui-même avec un niveau de difficulté "normal ». Les résultats de ces méthodes basées sur l'apprentissage actif (ILS, "Interactive Learning Strategies"), et plus spécifiquement sur les techniques de questionnement, d'investigation et d'autorésolution ("inquiry-based learning"(a)), sont significatifs en termes de consolidation et de structuration des connaissances.

D'autre part, le travail collaboratif en petits groupes, basé sur des échanges et des confrontations d'idées, permet à l'étudiant de découvrir que ce qu'il pense avoir compris ne l'a pas été de la même façon par ses camarades. Ceci génère questionnement et remise en cause.

Plus globalement, l'enseignement de la physique s'est enrichi ces dernières décennies de méthodes pédagogiques complémentaires. À côté des travaux en laboratoire, on peut citer le système des "clickers" (petits terminaux permettant de répondre à chaud à des questions du professeur et d'en tirer des statistiques, puis, par exemple, de former des sous-groupes d'étudiants pour résoudre
Tutorials in Introductory Physics and Homework Package

Encadré 1

\section{Lilian C. McDermott, Peter S. Schaffer and the Physics Education Group}

Pearson, 2 volumes (256 et 192 pages), 2002, $71,99 \$$.

Éditée en 1998, la première version avait été traduite en allemand et en espagnol.

Son principal auteur, Lilian McDermott, a été récompensée en 2001 par la médaille Oersted ${ }^{(*)}$.

Cet ouvrage rassemble un ensemble de concepts et de ressources pédagogiques utilisés dans de nombreuses universités aux États-Unis.

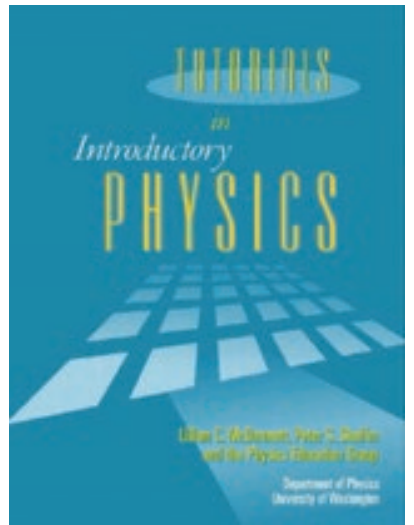

Les domaines abordés sont la mécanique, l'électricité et le magnétisme, les ondes, l'optique, et quelques sujets sélectionnés de physique moderne.

La deuxième édition de l'ouvrage, qui sortira prochainement, est enrichie de douze années supplémentaires de résultats expérimentaux et d'avancées conceptuelles, de nouveaux tutoriels et d'anciens tutoriels modernisés.

(*) Prix de l'Association américaine des professeurs de physique, créé en 1936, qui récompense des contributions notables dans le domaine de l'enseignement de la physique. Son nom vient du physicien danois Hans Christian Ersted. Lilian McDermott a été la deuxième femme à recevoir ce prix depuis sa création. 
$>>$

ensemble la question), les exercices en ligne avec retour immédiat, les vidéos préalables de découverte du cours, les techniques de résolution de problèmes coopératifs comme celles de l'Université du Minnesota [3]...

\section{Q : Passons maintenant à la méthode elle-même, les "Tutorials". \\ En quoi consistent-ils?}

PH : Nous nous focalisons sur la physique générale ${ }^{(b)}$, car ce sont ces quelques concepts, relations et principes fondamentaux qui ne sont pas toujours bien compris par les étudiants entrant à l'université. Souvent, les étudiants n'ont pas conscience qu'ils ne maitrisent pas pleinement ces concepts, ce qui est un véritable risque pour leur apprentissage futur.

Les “Tutorials" viennent compléter les cours et les exercices donnés aux étudiants. Il s'agit d'un ensemble de modules aidant ces derniers à développer de façon méthodique une compréhension complète, approfondie et structurée des fondamentaux de physique.

Pour les étudiants, chaque module consiste en un atelier en groupe. Ils reçoivent un guide écrit précis, très structuré, qui repose sur le modèle "déclencher-confronterrésoudre ». Les étudiants doivent donc répondre en groupe à une suite de questions et de petits exercices qui peuvent être "piégeants " et qui se recoupent, mais avec de légères différences, ce qui oblige à revoir de façon détaillée chaque étape du raisonnement, réviser chaque relation, et finalement à " reconstruire " les concepts par eux-mêmes. Des devoirs individuels à la maison viennent compléter le dispositif.

\section{Q : À part le cours et les "Tutorials",} avez-vous d'autres rendez-vous avec les étudiants, comme des travaux dirigés ou bien des travaux pratiques?

PH : Nous n'avons pas de travaux dirigés systématiques avec chaque cours comme vous les avez en Europe, mais nous avons bien sûr des séances d'expérimentation en laboratoire où les étudiants se familiarisent avec la mise en évidence expérimentale des phénomènes physiques et la confirmation des théories, ce qui est à la base de la physique.

\section{Q : Comment s'intègrent les "Tutorials" dans le programme de l'université ?}

PH : Nous fonctionnons comme les cours, à raison d'une séance réservée aux "Tutorials" chaque semaine. Les classes d'environ 200 étudiants sont fragmentées en groupes de 25. Les groupes forment des sous-groupes de trois ou quatre personnes (fig. 1), dont le mode de constitution est libre. Nous avons exploré divers formats, par niveaux homogènes, ou au contraire très hétérogènes, ou bien imposé certains critères, sans que cela s'avère plus efficace. Nous avons donc décidé de ne pas interférer dans la constitution de ces groupes, sauf quand nos encadrants détectent des dysfonctionnements. Les quelques étudiants exceptionnels se voient proposés un parcours "honor" un peu différent.

Q : Comment déterminez-vous les sujets qui posent difficulté aux étudiants et donc les "Tutorials" à créer et à mettre en place?

PH : Bien entendu, nous travaillons beaucoup avec les enseignants. Ils connaissent bien les difficultés que rencontrent leurs étudiants. Mais cela peut être une connaissance transversale qui est trop faible, ou un acquis traité en cours plusieurs semaines ou années en arrière qui manque.

Il n'est pas toujours facile de détecter les idées préconçues derrière les erreurs des étudiants aux examens classiques, même si, comme aux États-Unis, ils ont lieu très régulièrement toutes les trois ou quatre semaines.

Aussi avons-nous systématisé une démarche d'analyse scientifique de pré et post-tests (avant et après les "Tutorials") qui balaient de nombreux sujets, abordés les années précédentes ou enseignés lors d'un cours récent. Les pré-tests sont réalisés en ligne le week-end, les post-tests sont intégrés dans les évaluations du cours co-écrits avec le professeur.

Nous élaborons soigneusement des batteries de questions, avec de nombreuses déclinaisons et formulations différentes pour un même sujet.Vous seriez étonné de voir ce qu'on peut détecter en posant une question a priori simple comme : "Deux ampoules sont raccordées à une même source d'énergie, l'une est plus éloignée que l'autre de la source ; l'une d'entre elles est-elle moins brillante ? ". Hélas, l'erreur est fréquente et assez prévisible. Et quand une erreur est systématique, elle recoupe parfois une autre incompréhension.

\section{Q : À quels types d'étudiants vous adressez-vous?}

PH: Tous! En pratique, nous nous adressons prioritairement aux étudiants de niveau "moyen ", autant dire une grande majorité, pour leur permettre de renforcer leur socle de connaissances. Cependant, nous remarquons que les bons étudiants, les plus motivés, apprécient beaucoup les “Tutorials", car c'est une façon pour eux de se tester et de discuter physique avec les copains !

$\mathrm{Au}$ début, certains professeurs avaient l'intuition que les "Tutorials" allaient surtout aider les élèves en grande difficulté ; mais avec le temps, ils découvrent que ce n'est pas le cas, que les “Tutorials" sont rarement trop faciles, loin de là, et qu'ils aident vraiment tout type d'étudiant.

Cependant, les étudiants qui n'ont pas su ou pu acquérir un niveau minimum de connaissances (étudiants ayant maitrisé tard la langue anglaise, provenant de secteurs défavorisés...) ne peuvent pas juste être aidés avec cette méthode. La remise à niveau doit être plus ciblée.

\section{Q : Une fois les groupes constitués, que se passe-t-il durant une séance de "Tutorials" ?}

PH : C'est là que se situe la nouveauté. Les étudiants réfléchissent en groupe et peuvent demander l'aide des encadrants présents, mais nous ne donnons pas de réponse toute faite pendant la séance, ni de corrigé à la fin. Nous pensons que le plus important, c'est le processus de réflexion autour des exercices, l'échange entre les étudiants et leur argumentation, voire leur autocritique, qui leur permet de savoir s'ils sont sur la bonne voie ou bien s'ils se trompent.

\section{Q : Qui sont les encadrants et que} font-ils concrètement pendant ces séances?

PH : Les encadrants sont pour la plupart des étudiants "post-graduate" (niveau master) ou en thèse dans un domaine de la physique. Certains d'entre eux sont déjà passés par notre système, mais d'autres le découvrent. Nous avons donc chaque semaine une formation préparatoire avec tous les encadrants pour les confronter au "Tutorial" qu'ils devront encadrer. Ils jouent donc le jeu d'être étudiant, car ils ne peuvent pas se contenter de lire le “Tutorial". Ils apprennent à guider, reformuler, sans donner d'indices trop évidents ou de réponses toutes faites, même si les étudiants les réclament parfois à corps et à 
cri ! Ils vont s'aider de phrases types comme "pouvez-vous m'expliquer votre raisonnement ? " ou " comment en êtes-vous arrivé à cette conclusion ?».

\section{Q : Les "Tutorials" se concentrent-ils} sur le cours qui a eu lieu juste avant?

PH : Non, pas forcément. Les "Tutorials" abordent l'ensemble des notions qui doivent être comprises par l'étudiant à l'issue du cours, mais il peut s'agir de connaissances vues des mois auparavant. Nous cherchons la mémorisation et la compréhension sur des temps longs, pour consolider l'apprentissage.

\section{Q : Réalisez-vous une évaluation à la fin} de ces séances?

PH : Non, car d'abord les "Tutorials" ne sont pas obligatoires. Ils sont proposés aux étudiants, qui ont le choix de les suivre ou non. Dans les faits, la plupart, sinon tous, viennent aux ateliers. Ensuite, l'important n'est pas que les étudiants sachent répondre aux mêmes questions que celles de l'atelier quelques minutes ou heures après qu'il soit terminé, mais bien l'ancrage et la mémorisation à long terme des concepts, ce que nous vérifions grâce aux post-tests. Nous obtenons des améliorations de performances, qui sont de l'ordre de 15\% de réponses justes lors du pré-test à $70-75 \%$ pour le post-test.

Q : Les "Tutorials" amènent-ils d'autres améliorations de l'apprentissage que la solidité des connaissances en physique?

PH : Bien entendu, nous espérons contribuer à améliorer la capacité à apprendre par soi-même et le potentiel de raisonnement autonome des étudiants, mais nous n'avons pas encore trouvé les mesures fiables pour les évaluer. Nous savons que les étudiants exposés aux "Tutorials" réclament leur mise en place pour des cours plus avancés, d'une part parce qu'ils en reconnaissent l'efficacité, mais aussi parce qu'ils ont appris à apprécier le travail en groupe. Par exemple, ils nous en ont réclamé pour accompagner leur apprentissage de la mécanique quantique, ce sur quoi nous travaillons actuellement. Nous avons aussi noté que la plupart des étudiants sont plus autonomes après plusieurs "Tutorials", et que la dépendance au professeur diminue. En tout cas, ils ont compris l'utilité du travail collaboratif, ce qui peut leur être utile dans leur future activité professionnelle.
Expérimentation de "Tutorials" à l'Université Pierre et Marie Curie

Sous l'impulsion d'un groupe d'enseignants motivés, et avec le soutien du directeur de la faculté de l'époque, Patrick Boissé, un projet de test et d'implémentation d'innovations pédagogiques fondées sur l'apprentissage interactif (méthodes ILS, "Interactive Learning Strategies") a démarré à l'Université Pierre et Marie Curie (UPMC) au printemps 2012 dans le département de physique. Les motivations pour innover provenaient avant tout du constat fait, en particulier lors des travaux dirigés, d'une relative passivité des étudiants.

\section{Qu'est-ce qui a été expérimenté ?}

- La méthode "think-pair-share" (réfléchir-discuter-restituer) utilisant un "clicker" (voir p. 35) pendant les cours. Avant le cours, les étudiants doivent le lire et réaliser des recherches personnelles. Le temps du cours est animé avec des questions pour tester leur apprentissage. Le "clicker" permet d'évaluer les réponses avant et après les discussions en sous-groupes. À l'issue de la question, l'enseignant ne détaille que les points incompris, s'ils subsistent.

- La méthode "peer-to-peer" (pair à pair) via les "Tutorials" à la place de certains travaux dirigés, en particulier en première année de licence (L1).

La venue à l'UPMC en 2012 d'un professeur américain, Alexander Rudolph, a permis à cette équipe de se former aux méthodes développées par l'Université de Washington, dont les "Tutorials" en mécanique ont été traduits.

Le premier semestre de cette expérimentation a été résumé dans un article de recherche [6] Les cours de L1 de mécanique et de L2 d'électromagnétisme sont ceux qui ont été concernés par cette expérimentation.

\section{Témoignages sur l'expérimentation}

Les résultats indiquent que les méthodes ILS conduisent à une amélioration significative d'un facteur 2 ou plus de la compréhension des concepts de base par les étudiants. Avec ces méthodes, les étudiants deviennent plus actifs, renforcent le travail collaboratif, et prennent un meilleur contrôle de leur propre apprentissage.

En conséquence, une nouvelle maquette de l'unité d'enseignement (UE) mécanique, intégrant les principes de l'enseignement interactif, a été réalisée en 2013/2014. D'autres UE ont rejoint le mouvement, comme la thermodynamique.

Michael Joyce, responsable de l'UE de mécanique en 2012, intimement convaincu par l'efficacité de ces méthodes, nous donne son avis sur les trois clefs du succès de I'implémentation des techniques ILS comme celles mises en place à I'UPMC [6] :

- un usage régulier de la méthode du "clicker", car le "retour sur investissement " (motivation et apprentissage des étudiants) est rapide et excellent ;

- un bon équilibre entre les cours, les "Tutorials" et les travaux dirigés classiques, à penser en fonction du niveau des étudiants et de la motivation des professeurs.

Il est indispensable que les formateurs aux "Tutorials" soient motivés et surtout formés, car cette méthode est éloignée des fonctionnements habituels. La formation doit en particulier porter sur deux points : expliquer la méthode aux étudiants, assez déroutante pour certains, et maintenir leur curiosité tout au long du "Tutorial".

Yannis Zouganelis, qui a testé à la fois les "Tutorials" et la méthode "think-pair-share", en a apprécié les bénéfices, "en particulier lors des cours magistraux où la méthode des clickers permet d'aller assez loin sur les concepts ». II regrette cependant que «les Tutorials laissent peu de place à l'apprentissage des techniques de calcul ».

\section{Les résultats de l'expérimentation}

L'étude publiée [7] montre des résultats très positifs des méthodes ILS sur l'apprentissage des concepts, les résultats aux examens finaux, et les relations entre enseignants et étudiants, grâce à une amélioration de la motivation et de l'assiduité de ces derniers.

Cette étude a été cependant limitée à une seule année d'expérimentation.

Brahim Lamine a participé à l'expérimentation de 2012 et poursuit l'utilisation des méthodes "think-pair-share" en amphi à l'université Paul Sabatier de Toulouse, dans un contexte de massification des effectifs. L'analyse complète d'une expérimentation menée en 2014-2015 a été effectuée [8]. 
$>>$

$\mathbf{Q}:$ Avez-vous rencontré des résistances au début de la mise en place de votre méthodologie?

PH : Je suis arrivée dans l'équipe quelques années après le démarrage des "Tutorials". Ce que j'ai constaté à l'époque, et que je constate encore parmi les étudiants des premières années, c'est que ceux-ci sont habitués à recevoir la réponse toute faite par le professeur, et n'entrevoient pas l'intérêt d'y réfléchir par eux-mêmes.

Certains étudiants n'aiment pas travailler en groupe, d'autres pensent que travailler avec des étudiants qui sont plus lents dans leur travail va les ralentir, et enfin quelquesuns restent persuadés qu'ils ont tout compris. Mais ils déchantent après plusieurs "Tutorials" ! Globalement, les résistances disparaissent après quelques séances.

$\mathbf{Q}$ : Depuis combien de temps avez-vous mis en place ces nouvelles méthodes d'enseignement?

PH : Lilian McDermott en est à l'origine, il y a plus de trente ans [4]. Elle a été pionnière dans ce domaine qui a progressivement essaimé dans de nombreuses autres universités américaines. Elle a créé le groupe de recherche sur l'enseignement de la physique à l'Université de Washington dans les années 1980. Elle a lancé l'ouvrage Tutorials in Introductory Physics (encadré 1, p. 35), ainsi que les Physics by Inquiry ${ }^{(\mathrm{c})}$.

Q : Comment votre groupe s'intègre-t-il dans le paysage universitaire américain? PH: Nous avons une longue tradition de formation des jeunes, et encadrons actuellement sept étudiants en thèse. Nous avons conduit plus de 25 thèses depuis les années 1980, et nos étudiants ont par la suite formé d'autres groupes dans d'autres universités avec lesquelles nous sommes en contact. Ces dernières années ont donc vu naitre une dizaine de groupes de recherche sur l'enseignement de la physique, en particulier dans les universités du Maryland, de l'Illinois, du Michigan, de l'Ohio, l'Université Colorado Boulder ou encore Cornell. Ce sont de grands pôles, où vous pouvez passer une thèse en physique avec une spécialisation " recherche en éducation de la physique ». Nous sommes maintenant en concurrence avec eux pour les meilleurs étudiants !

Plus largement, il y a maintenant un réseau américain constitué autour de la méthode des "Tutorials", appliquée dans de très nombreuses universités, dont la plupart n'ont d'ailleurs pas de groupe de recherche sur l'enseignement. Plus globalement, nous espérons contribuer à ce que plus d'étudiants en physique aient la fibre de l'enseignement, ce qui n'est pas une tâche facile.

\section{Q : Comment font ces universités} pour se former à vos méthodes ?

PH : Nous accueillons les enseignants et chercheurs à l'université de Washington pendant plusieurs semaines. Ils participent aux réunions avec les encadrants et aux séances de “Tutorials". Une fois qu'ils comprennent comment cela fonctionne, ils peuvent retourner dans leurs universités respectives et nous continuons à interagir avec eux régulièrement.

\section{Q : Votre méthode commence-t-elle à se diffuser en Europe?}

PH : Je vais tous les ans à la conférence du GIREP (Groupe international de recherche sur l'enseignement de la physique). Nous avons des relations avec des groupes de recherche dans diverses universités en Irlande, Écosse, Finlande, Espagne, Italie et Allemagne, qui traitent de l'enseignement de la physique dans le supérieur.L'Université de Hambourg utilise beaucoup les "Tutorials" pour former les futurs ingénieurs ; elle a assuré la traduction et la parution de notre ouvrage.

En France, la recherche en éducation à la physique est bien développée, mais plutôt orientée sur le secondaire [5]. Cependant, une expérimentation des plus intéressantes a été initiée en 2012 à l'Université Pierre et Marie Curie avec la méthode des “Tutorials" (voir l'encadré 2, p. 37).
Les cours magistraux classiques ne permettent que rarement de remettre en cause des préjugés bien ancrés...

Le travail collaboratif en petits groupes, basé sur des échanges et des confrontations d'idées, permet à l'étudiant de découvrir que ce qu'il pense avoir compris ne l'a pas été de la même façon par ses camarades.

Ceci génère questionnement et remise en cause.

(a) L" "inquiry-based learning", souvent traduit en français par « enseignement par investigation ", suppose que l'étudiant devienne actif dans son apprentissage. Ce type d'enseignement est particulièrement mis en avant dans l'éducation des sciences, car il s'apparente à la démarche suivie par les scientifiques. Il implique un changement profond du rôle du professeur, qui passe de l'enseignant partageant son savoir à l'accompagnateur de la démarche et de la réflexion de l'étudiant. En France, la " démarche d'investigation ", généralisée dans l'enseignement des sciences au lycée, est une illustration de l'intégration de ces nouvelles pédagogies dans l'enseignement secondaire [5].

(b) Pour découvrir les "Tutorials" sur les $2^{\mathrm{e}}$ et $3^{\mathrm{e}}$ lois de Newton au niveau équivalent L1, et des conseils d'utilisation de l'Université de Colorado Boulder https://cutt.ly/physport-methods

(c) "Physics by inquiry" est un ensemble de modules basés sur des montages de laboratoires, pour préparer les enseignants du primaire et du secondaire à enseigner les sciences de façon pratique et à en dégager les concepts utiles pour leur enseignement.

\section{Références}

$1 \cdot$ www.aps.org/programs/education/undergrad/jtupp.cfm

2- https://phys.washington.edu/tutorials-introductory-physics

3• http://groups.physics.umn.edu/physed/Research/CGPS/CGPSintro.htm

4 L.C. McDermott, Oersted Medal Lecture 2001, "Physics Education Research - The Key to Student Learning", Am. J. Phys. 69 (2001) 1127-1137.

5• www.education.gouv.fr/cid54197//-enseignement-des-sciences.html

6• A.L. Rudolph et al., "Introduction of interactive learning into French university physics classrooms", Phys. Rev. Spec. Top. 10.010103 (2014).

7• http://video.upmc.fr/differe.php?collec=E_rencontres_pedagogiques_2017\&video=7

8• www.fsi.univ-tlse3.fr/enseignements-interactifs-bilan-de-I-experience-de-la-fsi-633019.kjsp 